\title{
A Note on Unlinking Numbers of Montesinos Links
}

\author{
K. MOTEGI
}

ABSTRACT. Let $K$ (resp. $L$ ) be a Montesinos knot (resp. link) with at least four branches. Then we show that the unknotting number (resp. unlinking number) of $K$ (resp. $L$ ) is greater than 1 .

\section{INTRODUCTION}

The unknotting number (resp. unlinking number) of a knot $K$ (resp. link $L$ ) in $S^{3}, u(K)$ (resp. $u(L)$ ) is the minimum number of crossing changes needed to create the unknot (resp. unlink). The minimum being taken over all possible sets of changes in all possible presentations of $K$ (resp. $L$ ).

These numbers are very intuitive invariant and not easy to calculate. In [14], Scharlemann proved that unknotting number one knots are

1991 Mathematics Subject Classification: $57 \mathrm{M} 25$

Servicio publicaciones Univ. Complutense. Madrid, 1996.

Research partially supported by Grant-in-Aid for Encouragement of Young Scientists 06740083, The Ministry of Education, Science and Culture and Nihon University Research Grant B94-0025. 
prime. An alternative proof was given by Zhang [18]. The analogous result for links (i.e., unlinking number one links are prime) was proved by Eudave-Muñoz [3] and Gordon-Luecke [4] in different methods. For two bridge knots, Kanenobu-Murakami [6] determined two bridge knots with unknotting number one. Later Kohn [7] determined two bridge links with unlinking number one. Recently Menasco [9] determined the unknotting (resp. unlinking) number of torus knots (resp. torus links). A survey of methods of calculation of unknoting numbers is given by Nakanishi [13].

In this paper, we study unknotting numbers (resp. unlinking numbers) of Montesinos knots (resp. Montesinos links).

Let $M\left(e ;\left(\alpha_{1}, \beta_{1}\right), \ldots,\left(\alpha_{r}, \beta_{r}\right)\right)$ be a Montesinos knot or link with $r$ branches (see Figure 1), where a box $\alpha_{i}, \beta_{i}$ stands for a so-called "rational tangle" of type $\left(\alpha_{i}, \beta_{i}\right)([11],[12],[19]$ and [2]).

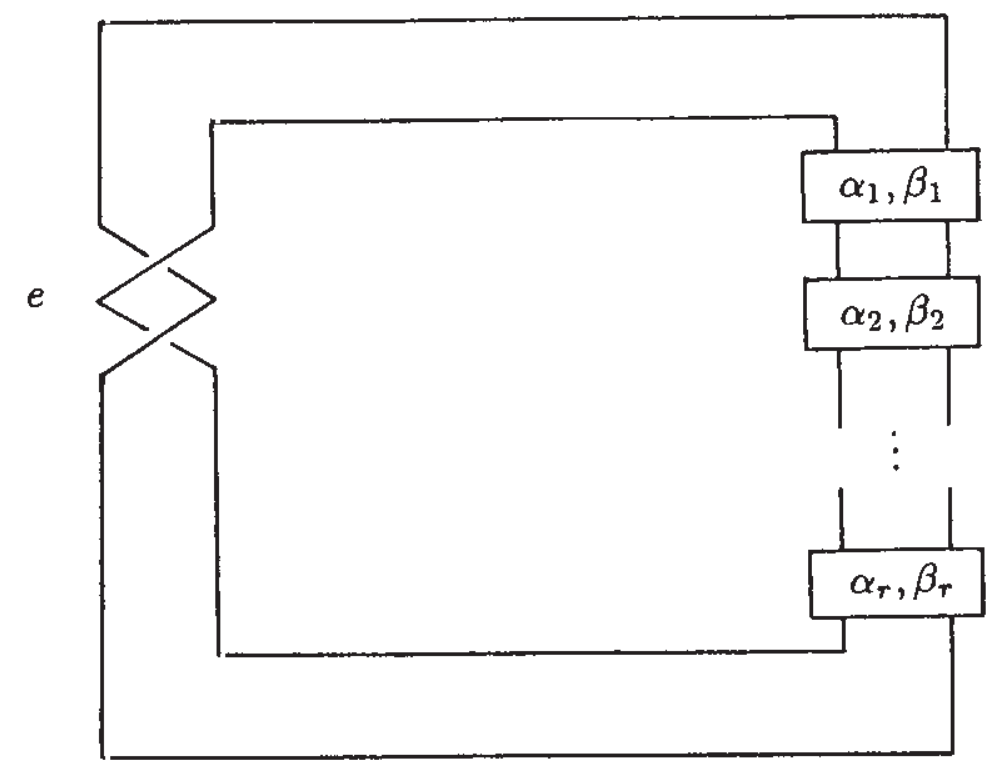

Figure 1

In the following we assume that $\alpha_{i}>1$. (If for some $i, \alpha_{i}=1$, then the knot or link would have a simpler form.) 
Montesinos knot with $r \leq 3$ can have unknotting number one. For example $8_{20}=M(1 ;(2,1),(3,1),(3,2))$ has unknotting number one (see Figure 2).

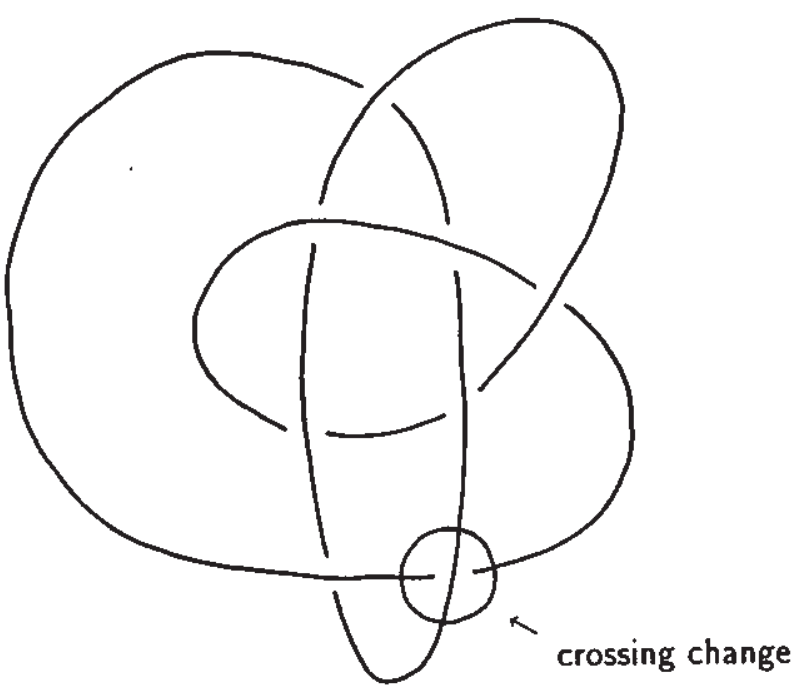

$$
8_{20}=M(1 ;(2,1),(3,1),(3,2))
$$

Figure 2

On the other hand if $r \geq 4$, we prove the following.

Theorem 1.1. Let $K=M\left(e ;\left(\alpha_{1}, \beta_{1}\right), \ldots\left(\alpha_{r}, \beta_{r}\right)\right)$ be a Montesinos knot with $r \geq 4$. Then $u(K) \geq 2$.

The two components Montesinos link $L=M(0 ;(3,1),(3,-1)$, $(5,2)$ ) illustrated by Figure 3 has $u(L)=1$.

If $r \geq 4$, we have:

Theorem 1.2. Let $L=M\left(e_{;}\left(\alpha_{1}, \beta_{1}\right), \ldots,\left(\alpha_{r}, \beta_{r}\right)\right)$ be a Montesinos link with $r \geq 4$. Then $u(L) \geq 2$. 
The present pronfs of Theorems 1.1 and 1.2 follow the same philosophy of [6], [7], [18] and [4], except for the case where $L$ has more than two components (Proposition 4.6).

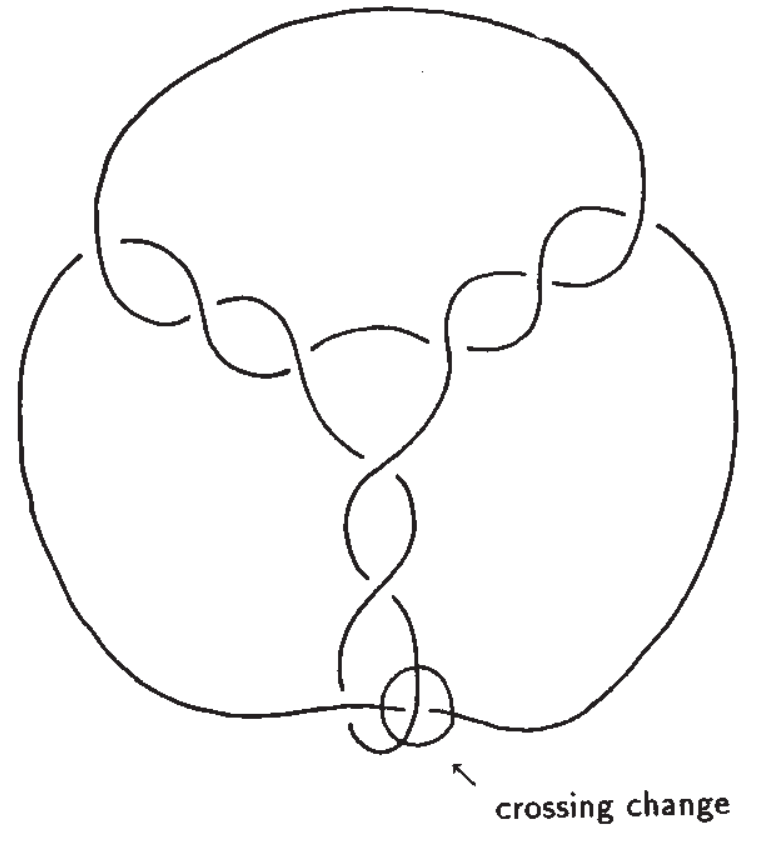

$$
L=M(0 ;(3,1),(3,-1),(5,2))
$$

Figure 3

\section{PRELIMINARIES}

Let $k$ be a knot in the interior of an orientable 3-manifold $M$. Let $N(k)$ be a tubular neighborhood of $k$ in $M$. For the isotopy class (slope) $\alpha$ of an essential simple closed curve on $\partial N(k), M(k ; \alpha)$ denotes the manifold obtained from $M$ by $\alpha$-surgery on $k$, i.e., the result of attaching a solid torus $V$ to $M$-int $N(k)$ by identifying $\partial V$ with $\partial N(k)$ so that $\alpha$ bounds a disk in $V$. If $\alpha$ and $\beta$ are two slopes on $\partial N(k)$, then $\Delta(\alpha, \beta)$ denotes their minimal geometric intersection number.

If $K$ (resp. $L$ ) is a knot (resp. link) in $S^{3}$, we use $M_{K}$ (resp. $M_{L}$ ) to denote the two-fold branched covering of $S^{3}$ branched over the knot $K$ (resp. the link $L$ ). 
Lemma 2.1 ([11], [8] and [7]). (1) Let $K$ be a knot in $S^{3}$ with $u(K)=1$, then $M_{K}$ is homeomorphic to $S^{3}(k ; \gamma)$ for some knot $k \subset S^{3}$ and $\gamma$ with $\Delta(\gamma, \mu)=2$, where $\mu$ is a meridian slope of $k$.

(2) Let $L$ be a two components link in $S^{3}$ with $u(L)=1$, then $M_{L}$ is homeomorphic to $S^{2} \times S^{1}(k ; \gamma)$ for some knot $k \subset S^{2} \times S^{1}$ and $\gamma$ with $\Delta(\gamma, \mu)$, where $\mu$ is a meridian slope of $k$.

Lemma 2.2 ([11], [12], [19], [2]). The two-fold branched covering of $S^{3}$ branched over a Montesinos knot or link $M\left(e ;\left(\alpha_{1}, \beta_{1}\right), \ldots\right.$, $\left.\left(\alpha_{r}, \beta_{r}\right)\right)$ is a Seifert fibred manifold with the 2-sphere $S^{2}$ as base, obstruction invariant $e$ and $r$ exceptional fibres of types $\left(\alpha_{i}, \beta_{i}\right)$.

Lemma 2.3 ([1], [10]). Let $k$ be a non-hyperbolic knot in $S^{3}$. If $S^{3}(k ; \gamma)$ is a Seifert fibred manifold over $S^{2}$ with at least four exceptional fibres, then $\Delta(\gamma, \mu)=1$.

Remark. In [10] it is also proved that if there are two such surgery slopes $\gamma_{1}$, and $\gamma_{2}$, then $\Delta\left(\gamma_{1}, \gamma_{2}\right) \leq 1$.

A 3-manifold $M$ is a cable on a manifold $M_{1}$, if $M=C \cup_{T} M_{1}$ where $C$ is a cable space [5], $\partial M \subset \partial C$ and $T=\partial C \cap \partial M_{1}$ is an incompressible torus in $M_{1}$.

Lemma 2.4 ([1, Theorems 0.5 and 0.6]). Let $M$ be a closed orientable 3-manifold and $k$ a knot in $M$. Assume that $M$-int $N(k)$ is irreducible and is neither a Seifert fibred manifold nor a cable on a (boundary-irreducible) Seifert fibred manifold. If $M\left(k ; \gamma_{1}\right)$ is a Seifert fibred manifold over $S^{2}$ with at least four exceptional fibres and $M\left(k ; \gamma_{2}\right)$ has a cyclic fundamental group, then $\Delta\left(\gamma_{1}, \gamma_{2}\right) \leq 1$.

In particular the above lemma implies,

Corollary 2.5 ([1]). Let $k$ be a hyperbolic knot in $S^{3}$. If $S^{3}(k ; \gamma)$ is a Seifert fibred manifold over $S^{2}$ with at least four exceptional fibres, then $\Delta(\gamma, \mu)=1$, where $\mu$ is a meridian slope of $k$.

\section{PROOF OF THEOREM 1.1}

Let $K=M\left(e ;\left(\alpha_{1}, \beta_{1}\right), \ldots,\left(\alpha_{r}, \beta_{r}\right)\right)$ be a Montesinos knot with $r \geq 4$. Assume for contradiction that $K$ has unknotting number one. 
From Lemma 2.1 (1), we see that $M_{K}$ (the two-fold branched covering of $S^{3}$ branched covering over $\left.K\right)$ is homeomorphic to $S^{3}(k ; \gamma)$ for some knot $k\left(\subset S^{3}\right)$ and $\gamma$ with $\Delta(\gamma, \mu)=2$, where $\mu$ is a meridian slope of $k$. Since $\mathrm{K}$ is a Montesinos knot with $r(\geq 4)$ branches, $M_{K}$ is a Seifert fibred manifold over $S^{2}$ with $r(\geq 4)$ exceptional fibres. Therefore Lemma 2.3 and Corollary 2.5 imply that $\Delta(\gamma, \mu)=1$, a contradiction. Hence $K$ cannot have unknotting number one.

\section{PROOF OF THEOREM 1.2.}

To prove Theorem 1.2, we divide into two cases : (1) the link $L$ has exactly two components, or (2) $L$ has more than two componentes.

First we consider the case (1).

Proposition 4.1. Let $L=M\left(e ;\left(\alpha_{1}, \beta_{1}\right), \ldots,\left(\alpha_{r}, \beta_{r}\right)\right)$ be a two components Montesinos link with $r \geq 4$. Then $u(L) \geq 2$.

We prepare some lemmas to prove this proposition.

Lemma 4.2. Let $k$ be a knot in $S^{2} \times S^{1}$. If $S^{2} \times S^{1-i n t N(k)}$ is reducible, then $k$ is a local knot, i.e., there exists a 3-ball $B^{3}$ in $S^{2} \times S^{1}$ such that $B^{3} \supset k$.

Proof. Let $\Sigma$ be an essential 2-sphere in $S^{2} \times S^{1}$-int $N(k)$. If $\Sigma$ separates $S^{2} \times S^{1}-\operatorname{int} N(k)$, then since $S^{2} \times S^{1}$ is prime it bounds a 3-ball in $S^{2} \times S^{1}$ containing $k$. Thus $k$ is a local knot.

If $\Sigma$ does not separate $S^{2} \times S^{1}$-int $N(k)$, then we take a simple loop $J$ in $S^{2} \times S^{1-\operatorname{int} N(k)}$ meeting $\Sigma$ transversely in a single point. The boundary $\Sigma^{\prime}$ of a tubular neighborhood of $\Sigma \cup J$ is a 2-sphere which separates $S^{2} \times S^{1}$ into $X_{1}=N(\Sigma \cup J)$ and $X_{2}=S^{2} \times S^{1}$-int $N(\Sigma \cup J)$. Since $S^{2} \times S^{1}$ is prime and $X_{1}$ is not a 3 -ball, $X_{2}(\supset k)$ is a 3-ball. Hence $k$ is a local knot in $S^{2} \times S^{1}$.

Lemma 4.3. Let $k$ be a local knot in $S^{2} \times S^{1}$. If $S^{2} \times S^{1}(k ; \gamma)$ is Seifert fibred, then $S^{2} \times S^{1}(k ; \gamma) \cong S^{2} \times S^{1}$. (In particular $S^{2} \times S^{1}(k ; \gamma)$ is not a Seifert fibred manifold over $S^{2}$ with at least four exceptional fibres for auy slope $\gamma$.) 
Proof. Since $k$ is local, $S^{2} \times S^{1}(k ; \gamma)$ has $S^{2} \times S^{1}$ as a connected summand. A reducible Seifert fibred manifold is homeomorphic to $S^{2} \times$ $S^{1}$ or $P^{3} \# P^{3}, P^{3}$ is a real projective space and the result follows.

In the following $S^{3}$ and $S^{2} \times S^{1}$ are not considered as lens spaces.

Lemma 4.4. Let $k$ be a knot in $S^{2} \times S^{1}$ such that $S^{2} \times S^{1}-i n t N(k)$ is a Seifert fibred manifold or a cable on a Seifert fibred manifold. Then $S^{2} \times S^{1}(k ; \gamma)$ cannot be a Seifert fibred manifold over $S^{2}$ with at least four exceptional fibres for any slope $\gamma$.

Proof. Suppose for contradiction that $S^{2} \times S^{1}(k ; \gamma)$ admits a Seifert fibration over $S^{2}$ with at least four exceptional fibres. Then the Seifert fibration is unique [5, VI.17] (because $S^{2} \times S^{1}(k ; \gamma)$ is not the double of a twisted I-bundle over the Klein bottle), and any incompressible torus is isotopic to a vertical one (i.e., a union of fibres) ([16]).

Case 1. $S^{2} \times S^{1}-\operatorname{int} N(k)$ is Seifert fibred.

In this case from [7, Lemma 4] we see that $k$ is a regular fibre in some Seifert fibration of $S^{2} \times S^{1}$. Since any Seifert fibration of $S^{2} \times S^{1}$ has $S^{2}$ as base with zero or two exceptional fibres, $S^{2} \times S^{1}$-int $N(k)$ is Seifert fibred over the disk $D^{2}$ with zero or two exceptional fibres. If the surgery slope $\gamma$ coincides with a regular fiber of $S^{2} \times S^{1}$-int $N(k)$, then the result $S^{2} \times S^{1}(k ; \gamma)$ is the 3 -sphere $S^{3}$ or a connected sum of two lens spaces, which cannot admit a Seifert fibration over $S^{2}$ with at least four exceptional fibres. If $\gamma$ is not a regular fibre of $S^{2} \times S^{1}$-int $N(k)$, then $S^{2} \times S^{1}(k ; \gamma)$ admits a Seifert fintation extending that of $S^{2} \times S^{1}-$ $\operatorname{int} N(k)$. Hence the result $S^{2} \times S^{1}\left(k_{\ddagger} \gamma\right)$ is Seifert fibred over $S^{2}$ with at most three exceptional fibres. It follows that $S^{2} \times S^{1}(k ; \gamma)$ cannot admit a Seifert fibration over $S^{2}$ with at least four exceptional fibres.

Case 2. $S^{2} \times S^{1}-\operatorname{int} N(k)$ is not Seifert fibred : $S^{2} \times S^{1}-\operatorname{int} N(k)$ is a cable on a (boundary-irreducible) Seifert fibred manifold.

Let $C\left(\subset S^{2} \times S^{1}\right.$-int $\left.N(k)\right)$ be the cable space and $M_{1}\left(\subset S^{2} \times S^{1}-\right.$ $\operatorname{int} N(k))$ the Seifert fibred manifold. Let $\mu$ be the slope of a meridian of $k$ in $S^{2} \times S^{1}$ and $\tau$ the slope of a regular fibre of the cable space $C$. 
Claim 4.5. $\Delta(\tau, \mu)=1$.

Proof of Claim 4.5. If $\tau=\mu(i . e ., \Delta(\tau, \mu)=0)$, then $C \cup N(k)$ ( $C S^{2} \times S^{1}$ ) and hence $S^{2} \times S^{1}$ has a lens space summand, a contradiction. If $\Delta(\tau, \mu) \geq 2$, then the Seifert fibration of the cable space $C$ can be extended to that of $C \cup N(k)$, which is boundary-irreducible. Since $M_{1}$ is also boundary-irreducible, $S^{2} \times S^{1}$ contains an incompressible torus. This is a contradiction.

It follows that $C \cup N(k)$ is a solid torus in $S^{2} \times S^{1}$, whose core is the exceptional fibre $f$ of the cable spce $C$. Thus we can regard $C \cup N(K)$ as a tubular neighborhood $N(f)$ of $f$ in $S^{2} \times S^{1}$.

If the surgery slope $\gamma$ coincides with $\tau$ (i.e., $\Delta(\gamma, \tau)=0$ ), then $C \cup_{\gamma} V$, where $V$ denotes the filling solid torus, has a lens space summand. This implies that $S^{2} \times S^{1}(k ; \gamma)$ has a lens space summand. Hence it cannot be a Seifert fibred manifold over $S^{2}$ with at least four exceptional fibres. Now we consider the case where the surgery slope $\gamma$ does not coincide with $\tau$. In this case the Seifert fibration of $C$ can be extended to that of $C \cup_{T} V$. Suppose that $\Delta(\gamma, \tau)=1$. Then $C \cup_{\gamma} V$ becomes a solid torus whose core is the exceptional fibre $f$ in the cable space $C$. Therefore $S^{2} \times S^{1}(k ; \gamma) \cong S^{2} \times S^{1}\left(f ; \gamma^{\prime}\right)$ for some slope $\gamma^{\prime}$ on $\partial N(f)$. Since the exterior $S^{2} \times S^{1}-\operatorname{int} N(f)=M_{1}$ is Seifert fibred, we can conclude that $S^{2} \times S^{1}\left(f ; \gamma^{\prime}\right)$ cannot have a Seifert fibration over $S^{2}$ with at least four exceptional fibres by Case 1 . Let us assume that $\Delta(\gamma, \tau) \geq 2$. In this case $C \cup_{\gamma} V$ admits a Seifert fibration over $D^{2}$ with just two exceptional fibres by extending the Seifert fibration of $C$. Since both $M_{1}$ and $C \cup_{\gamma} V$ are boundary-irreducible, $S^{2} \times S^{1}(k ; \gamma)$ contains the incompressible torus $\partial M_{1}$, which can be assumed to be vertical by isotoping the Seifert fibration. If $C U_{\gamma} V$ is not a twisted I-bundle over the Klein bottle, then the Seifert fibration is unique up to isotopy ([5, VI.18.Theorem]). Therefore the Seifert fibration of $C \mathrm{U}_{\gamma} V$ which extends that of $C$ is isotopic to the Seifert fibration of $C \cup_{\gamma} V$ which is the restriction of that of $S^{2} \times S^{1}(k ; \gamma)$. Hence $S^{2} \times S^{1}-\operatorname{int} N(k)=C \cup M_{1}$ is Seifert fibred, a contradiction. We assume that $C U_{\gamma} V$ is a twisted I-bundle over the Klein bottle. Then it has just two Seifert fibrations up to isotopy ([17]) : the extended Seifert fibration of the cable space $C$ or a Seifert fibration over Möbius band with no exceptional fibre. In the 
first case the above argument implies that $S^{2} \times S^{1}$-int $N(k)=C \cup M_{1}$ is Seifert fibred, a contradiction. In the latter case $S^{2} \times S^{1}(k ; \gamma)$ is Seifert fibred over a non-orientable surface, and hence cannot admit a desired Seifert fibration.

Proof of Proposition 4.1. Let $L=M\left(e ;\left(\alpha_{1}, \beta_{1}\right), \ldots,\left(\alpha_{r}, \beta_{r}\right)\right)$ be a two components Montesinos link with $r \geq 4$. Assume for contradiction that $u(L)=1$. From Lemma 2.1(2), we see that the two-fold branched covering $M_{L}$ of $S^{3}$ branched over $L$ is homeomorphic to $S^{2} \times S^{1}(k ; \gamma)$ for some knot $k$ in $S^{2} \times S^{1}$ and $\gamma$ with $\Delta(\gamma, \mu)=2$, where $\mu$ is a meridian slope of $k$ in $S^{2} \times S^{1}$. Since $L$ is a Montesinos link with $r(\geq 4)$ branches, $M_{L}$ is a Seifert fibred manifold over $S^{2}$ with $r(\geq 4)$ exceptional fibres. If $S^{2} \times S^{1}-\operatorname{int} N(k)$ is reducible, then by Lemma $4.2, k$ is a local knot and $S^{2} \times S^{1}(k ; \gamma)$ cannot be a Seifert fibred manifold over $S^{2}$ with at least four exceptional fibres by Lemma 4.3. So we may assume $S^{2} \times S^{1}-$ $\operatorname{int} N(k)$ is irreducible. Suppose that $S^{2} \times S^{1}$-int $N(k)$ is Seifert fibred manifold or a cable on a Seifert fibred manifold. In this special case, by Lemma $4.4 S^{2} \times S^{1}(k ; \gamma)$ is not a desired Seifert fibred manifold. It follows from Lemma 2.4 that we have $\Delta(\gamma, \mu) \leq 1$, this is a contradiction. Therefore $u(L) \geq 2$.

As for the case (2): the link $L$ has more than two components, we can prove the following proposition.

Proposition 4.6. Let $L=M\left(e ;\left(\alpha_{1}, \beta_{1}\right), \ldots,\left(\alpha_{r}, \beta_{r}\right)\right)$ be a Montesinos link with mone than two components. Then $u(L) \geq 2$.

Proof. In the following we use indices modulo $r$. Let $C_{i, 1}$ and $C_{i, 2}$ be parallel arcs in $L$ connecting two rational tangles $\alpha_{i}, \beta_{i}$ and $\underline{\alpha_{i+1}, \beta_{i+1}}$ (see Figure 4). 


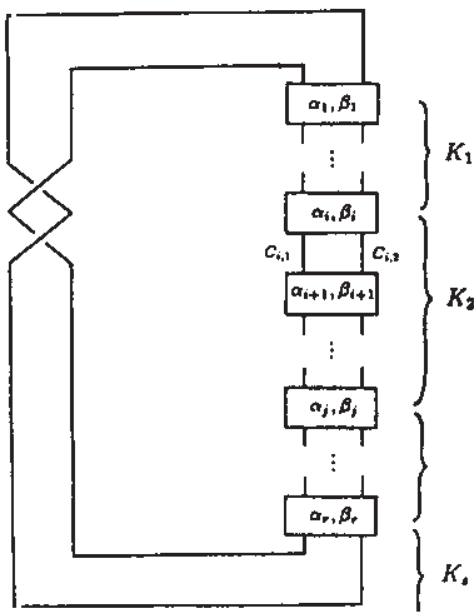

Figure 4

Claim 4.7. For each $i$, two arcs $C_{i, 1}$ and $C_{i, 2}$ are contained in the same component of $L$.

Proof of Claim 4.7. If for some $j, C_{j, 1}$ and $C_{j, 2}$ are contained in distinct components of $L$, then $C_{j, 1}$ and $C_{j+1, k}(k=1$ or 2$)$ are contained in the same component, and hence $C_{j, 2}$ and $C_{j+1,3-k}$ are also contained in the same component. Thus $C_{j+1, i}$ and $C_{j+1,2}$ are contained in distinct components. Inductively we can observe that for each $i, C_{i, 1}$ and $C_{i, 2}$ are contained in distinct components. Hence $L$ has exactly two components, a contradiction.

By Claim 4.7, components of $L$ are positioned as in Figure 4, i.e., components $K_{1}, \ldots, K_{s}$ of $L$ appear in clockwise order.

Suppose for contradiction that $L$ has unlinking number one. There are two possibilities: a crossing change on the same component of $L$ converts $L$ into the unlink or a crossing change on distinct components of $L$ converts $L$ into the unlink.

Suppose that a crossing change on a component $K_{i}$ transforms $L$ into a trivial link. Then since the link type of $K_{i+1} \cup K_{i+2}$ is not changed under the crossing change, the sublink $L^{\prime}=K_{i+1} \cup K_{i+2}$ is trivial. Next we consider the case where a crossing change on distinct components $K_{i}$ and $K_{j}(i \neq j)$ converts $L$ into a trivial link. Then we can take a component $K_{j^{*}}\left(=K_{j-1}\right.$ or $\left.K_{j+1}\right)$ so that $K_{j^{*}} \neq K_{i}$. Since the crossing change does not change the link type of $K_{j} \cup K_{j^{-}}$, the sublink $L^{\prime}=K_{j} \cup K_{j *}$ is a trivial link. 


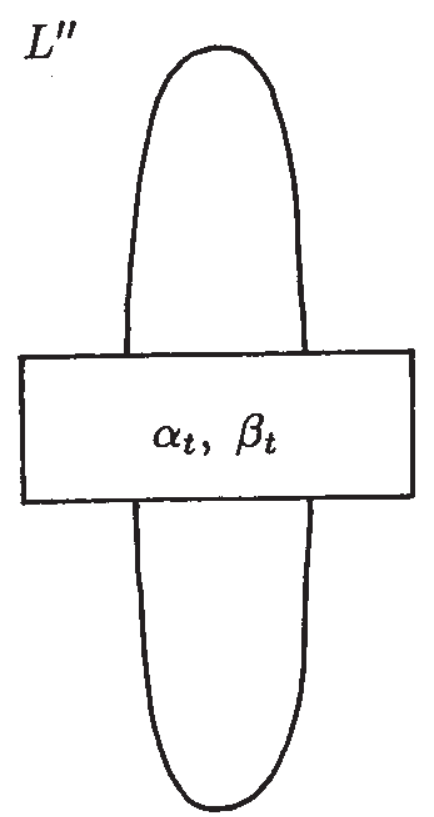

Figure 5

In any case each component of $L^{\prime}$ intersects a rational tangle $\alpha_{t}, \beta_{t}$ for some $t(1 \leq t \leq r)$. Therefore $L^{\prime}$ has a connected summand $L^{\prime \prime}$ given by Figure 5 .

Since $\alpha_{t}>1$, the factor link $L^{\prime \prime}$ is non-trivial (see [15]). Hence $L^{\prime}$ is also non-trivial, a contradiction. This completes the proof of Proposition 4.6.

Theorem 1.2 follows from Propositions 4.1 and 4.6.

\section{EXAMPLES}

Example 5.1. Let $K$ be a Montesinos knot $M(0 ;(4,3),(3,2)$, $(5,2),(5,-4))$ (see Figure 6$)$. Then by changing the indicated crossings in Figure 6, we obtain a trivial knot. Thus $u(K) \leq 2$. On the other hand Theorem 1.1 implies that $u(K) \geq 2$ and hence $u(K)=2$ 


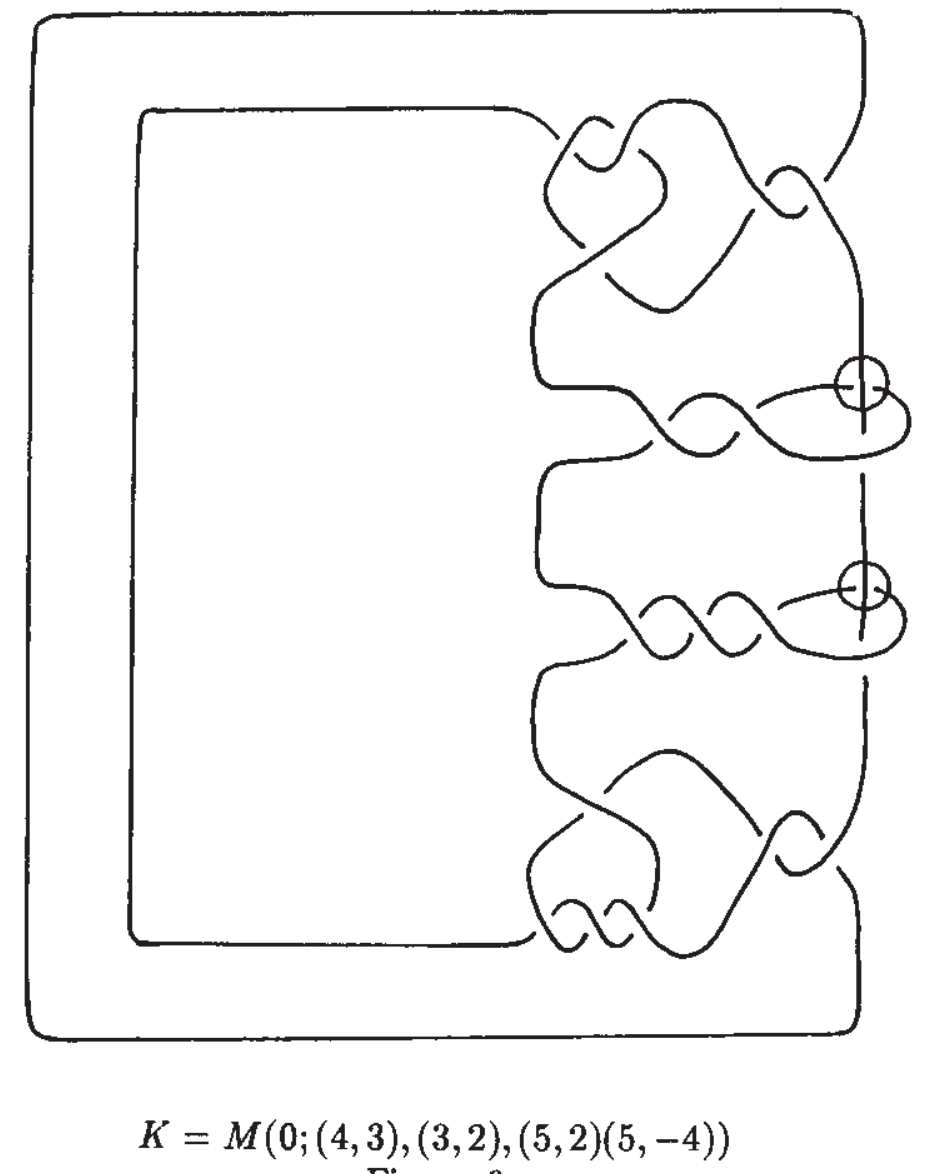

Figure 6

Example 5.2. Let $L$ be a Montesinos link $M(0 ;(5,-2),(5,2)$, $(5,-2),(5,2))$ with two components $K_{1}$ and $K_{2}$ (see Figure 7 ). If we change crossings at $\left\{p_{1}, p_{2}\right\}$ or $\left\{q_{1}, q_{2}\right\}$, we obtain a trivial link. Thus $u(L) \leq 2$. Hence we see that $u(L)=2$ by Theorem 1.2 .

We note that the crossing change at $p_{i}(i=1,2)$ is a crossing change on $K_{1}$ and the crossing change at $q_{i}(i=1,2)$ is a crossing change on $K_{1}$ and $K_{2}$. 


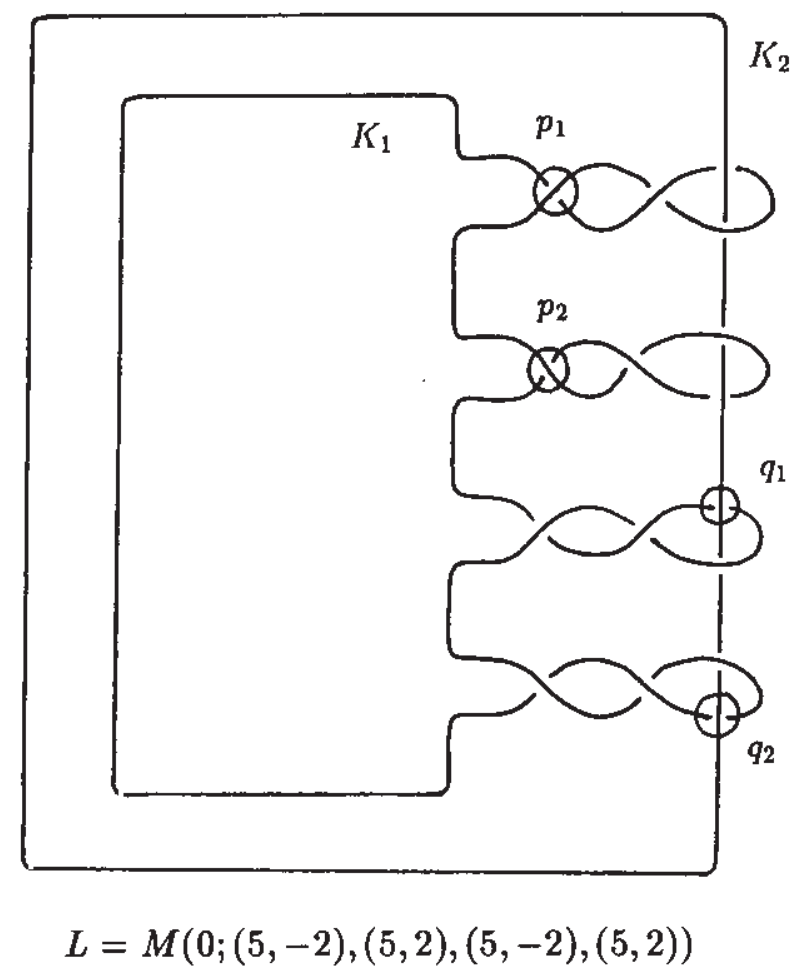

Figure 7

Acknowledgements I would like to thank Yasutaka Nakanishi for proving Proposition 4.6 and giving me Examples 5.1 and 5.2, and to thank Yoshiaki Uchida for useful conversations.

\section{References}

[1] Boyer, S. and Zhang, X., The semi-norm and Dehn filling, (preprint). [2] Burde, G. and Zieschang, H., Knots, de Gruyter Studies in Mathematics, no.5, Walter de Gruyter, Berlin, 1985.

[3] Eudave-Muñoz, M.,Primeness and sums of tangles, Trans. Amer. Math. Soc. 306 (1988), 773-790.

[4] Gordon, C.McA. and Luecke, J., Links with unlinking number one are prime, Proc. Amer. Math. Soc. 120 (1994), 1271-1274.

[5] Jaco, W., Lectures on three manifold topology, CBMS Regional Conference Series in Math. 43, Amer. Math. Soc., 1980. 
[6] Kanenobu, T. and Murakami, H., Two-bridge knots with unknotting number one, Proc. Amer. Math. Soc. 98 (1986), 499-502.

[7] Kohn, P., Two-bridge links with unlinking number one, Proc. Amer. Math. Soc. 113 (1991), 1135-1147.

[8] Lickorish, W.B.R., The unknotting number of a classical knot, Contemp. Math., vol. 44, Amer. Math. Soc., Providence, RI, 1985, pp. 117-121.

[9] Menasco, W., The Bennequin-Milnor unknotting conjectures, C. R. Acad. Sci. Paris, Série I 318 (1994), 831-836.

[10] Miyazaki, K. and Motegi, K., Seifert fibred manifolds and Dehn surgery, (to appear in Topology).

[11] Montesinos, J.M., Surgery on links and double branched coverings of $S^{3}$, Ann. of Math. Stud., no. 84, Princeton Univ. Press, Princeton, NJ, 1975, pp. 227-259.

[12] Montesinos, J.M., Variedades de Seifert que son recubridadores cíclicos ramificados de dos hojas, Bol. Soc. Mat. Mex. 18 (1973), $1-32$.

[13] Nakanishi, Y., A note on unknotting number, Math. Sem. Notes, Kobe Univ. 9 (1981), 99-108.

[14] Scharlemann, M., Unknotting number one knots are prime, Invent. Math. 82 (1985), 37-55.

[15] Schubert, H., Knoten mit zwei Brücken, Math. Zeit. 66 (1956), 133-170.

[16] Waldhausen, F., Eine Klasse von 9-dimensionalen Mannigfaltigkeiten I, II, Invent. Math. 3, 4 (1967), 308-333, 87-117.

[17] Wang, S. and Wu, Y.-Q., Covering invariants and cohopficity of 3-manifold groups, Proc. London Math. Soc. 68 (1994), 203-224.

[18] Zhang, X., Unknotting number one knots are prime : a new proof, Proc. Amer. Math. Soc. 113 (1991), 611-612.

[19] Zieschang, H., Classification of Montesinos knots, Proc. Leningrad 1982, Lect. Notes in Math., vol. 1060, 378-389, Springer-Verlag, 1984. 